\title{
Magnetoelectric properties of magnetite thin films $\uparrow$
}

\author{
J S-Y Feng§, R D Pashley $\ddagger$ and M-A Nicolet \\ California Institute of Technology, Pasadena, California 91125, USA
}

Received 28 June 1974, in final form 5 August 1974

\begin{abstract}
Resistivity, DC Hall effect and transverse magnetoresistance measurements were made on polycrystalline thin films of magnetite $\left(\mathrm{Fe}_{3} \mathrm{O}_{4}\right)$ from $104 \mathrm{~K}$ to room temperature. The Verwey transition is observed at $T_{\mathrm{V}}=123 \mathrm{~K}$, about $4 \mathrm{~K}$ higher than reported for bulk magnetite. The ordinary and extraordinary Hall coefficients are negative over the entire temperature range, consistent with negatively charged carriers. The extraordinary Hall coefficient exhibits a $\rho^{1 / 3}$ dependence on the resistivity above $T_{\mathrm{V}}$ and a $\rho^{2 / 3}$ dependence below $T_{\mathrm{v}}$. The magnetoresistance is negative at all temperatures and for all magnetic field strengths. The planar Hall effect signal was below the sensitivity of the present experiment.
\end{abstract}

\section{Introduction}

Since the discovery of the first lodestone in ancient Greece, magnetite has been an important magnetic material. Because all ferrites and garnets can be derived from magnetite by metal ion substitution, it is the prototypical ferrimagnetic compound. The electrical properties have been particularly illuminating since they led to the initial understanding of the ordering and exchange mechanisms in ferrites (Verwey et al 1947) and because magnetite was one of the first examples of an oxide in which a conductivity transition was observed (Weiss 1896).

Associated with the conductivity transition is a structural transformation (Bickford 1953), a specific heat anomaly (Millar 1929), and a change in the crystalline magnetic anisotropy ( $\mathrm{Li} \mathrm{1932).} \mathrm{The} \mathrm{conductivity} \mathrm{above} \mathrm{the} \mathrm{transition} \mathrm{temperature} \mathrm{is} \mathrm{a} \mathrm{low}$ mobility, high carrier density process and is believed to be due to localized electron states hopping to vacant sites (Heikes and Johnston 1959).

Previous measurements of the electrical properties have been performed on crystalline or ceramic samples (Bickford 1953, Lavine 1959, 1961, Kostopoulos and Theodossiou 1970, Kostopoulos 1972, Siemons 1970, Drabble et al 1971, Calhoun 1954 and Domenicali 1950). The conductivity has been measured from below $4 \mathrm{~K}$ to above the Curie temperature of $858 \mathrm{~K}$. The extraordinary Hall effect has been measured on bulk samples from $77 \mathrm{~K}$ to over $200^{\circ} \mathrm{C}$ (Lavine 1961, Kostopoulos and Theodossiou 1970). The ordinary Hall coefficient has been reported by three experimenters with conflicting results. Siemons (1970) reported a positive Hall coefficient, implying hole conductivity. On the other hand, Lavine (1959) and Kostopoulos and Theodossiou (1970) both reported negative Hall coefficients for other ferrites. Further evidence of electron con-

$\dagger$ Supported in part by the Office of Naval Research (L Cooper) and the Hoegsted Phim Memorial Fund.

¥ Present Address: Intel Corporation, Santa Clara, California, USA.

$\S$ Present Address: IBM Research, Yorktown Heights, New York, USA. 
duction is given by the negative Seebeck coefficients reported by Lavine (1959) and Constantin and Rosenberg (1972).

We present the results of resistivity, DC Hall effect and magnetoresistance measurements on polycrystalline thin film samples of magnetite.

\section{General considerations}

\subsection{Extraordinary Hall effect}

The Hall voltage in a ferromagnetic material depends separately on the magnetization and the external field. The Hall voltage has been found to follow

$$
V_{\mathrm{H}}=\left(R_{0} H_{\perp}+R_{1} M_{\perp}\right) I / t
$$

where $t$ is the sample dimension parallel to the applied magnetic field, $I$ is the current, $H_{\perp}$ is the component of the magnetic field perpendicular to the current and $M_{\perp}$ is the component of the magnetization perpendicular to the current. Note that $V_{H}$ depends only on the perpendicular components of $\boldsymbol{H}$ and $\mathbf{M} . \boldsymbol{R}_{0}$ is the ordinary Hall coefficient and $R_{1}$ is the extraordinary Hall coefficient. Experimentally, $R_{1}$ has been observed to be one to two orders of magnitude larger than $R_{0}$ (Pugh and Rostoker 1953).

Calculations have predicted that the extraordinary Hall coefficient should have a power law dependence on the resistivity

$$
R_{1} \propto \rho^{n}
$$

When the conductivity is due to non-localized d-band electrons, as in ferromagnetic metals, $R_{1}$ is proportional to $\rho^{2}$ (Karplus and Luttinger 1954). Measurements on ferromagnetic metals have shown that the actual dependence is very close to the predicted square law (Berger 1972).

\subsection{Magnetoresistance}

Semiclassical models predict that the apparent resistivity of a conductor should increase in the presence of a transverse magnetic field and it should be unchanged by a longitudinal field. Most materials have a positive transverse magnetoresistance and a much smaller longitudinal magnetoresistance (Putley 1968). Magnetite has been reported to have both positive and negative transverse magnetoresistance, depending on the magnitude of the applied field, the crystallographic orientation, the temperature, and the origin of the sample (Domenicali 1950, Zalesskii 1961, Kostopoulos 1970). The reported longitudinal magnetoresistance is of comparable magnitude (Domenicali 1950).

\subsection{Anisotropies}

A series of magnetic measurements revealed no evidence of measurable uniaxial, crystalline, or strain-induced anisotropies at room temperature. We observed no evidence of any anomalous effects in the present measurements at any temperature that might possibly be attributed to any magnetic anisotropies. 


\section{Experimental method}

\subsection{Sample preparation}

Magnetite samples were prepared by oxidation and reduction of iron thin films. Iron with greater than $99.96 \%$ purity was evaporated from alundum crucibles by $\mathrm{R} F$ induction heating in a vacuum of better than $10^{-6}$ Torr. The Corning 0211 glass substrates were outgassed and heated to $200^{\circ} \mathrm{C}$ during the deposition of the iron. The film thickness was determined from hysteresis loop tracer measurements (Humphrey 1967) with an estimated accuracy of $3 \%$. The thickness of this first iron film determines the thickness of the final $\mathrm{Fe}_{3} \mathrm{O}_{4}$ films.

Standard samples were $1 \mathrm{~cm}$ circular films. Photolithographic and etching techniques were used after the first iron deposition to define the shapes of other samples. The remaining iron was then oxidized at $400^{\circ} \mathrm{C}$ in $\mathrm{O}_{2}$ at 1 atm for 30 minutes to produce antiferromagnetic $\alpha-\mathrm{Fe}_{2} \mathrm{O}_{3}$. A second iron film was deposited at room temperature onto the $\alpha-\mathrm{Fe}_{2} \mathrm{O}_{3}$ and the samples were annealed in a vacuum of better than $10^{-7}$ Torr at $400^{\circ} \mathrm{C}$ for 10 hours to complete the formation of $\mathrm{Fe}_{3} \mathrm{O}_{4}$. The excess pure iron from the second deposition was removed by etching in dilute $\mathrm{HNO}_{3}$.

Films produced this way have been shown to be polycrystalline and strain-free at room temperature (Feng et al 1972). Any deviations from stoichiometry should result in films that are slightly iron-rich. In contrast, bulk samples could be iron-deficient because they require sintering or annealing at over $1000^{\circ} \mathrm{C}$ (Turner 1972, Smiltens 1952), and must usually be inspected for $\alpha-\mathrm{Fe}_{2} \mathrm{O}_{3}$ or $\gamma-\mathrm{Fe}_{2} \mathrm{O}_{3}$ content (Smiltens 1952).

Samples with three different shapes were used in these experiments. Two-point and four-point resistivity measurements were performed on the standard circular samples. A linear sample with contacts at the ends to supply current and a separate pair of contacts to sense the voltage was used for resistivity and magnetoresistance measurements. This sample was $0.5 \mathrm{~mm} \times 7 \mathrm{~mm}$ with $0.1 \mathrm{~mm}$ voltage sensing arms separated by about $3 \mathrm{~mm}$. A circular sample with four nearly equally spaced peripheral contacts was used for resistivity, magnetoresistance and Hall effect measurements using the method described by van der Pauw $(1958,1959)$. This sample was $2 \mathrm{~mm}$ in diameter with contact pads connected by $0.15 \mathrm{~mm}$ segments.

Typical samples are shown in the top of figure 1. Also shown in the inset is a transmission electron micrograph of a carbon replica showing the smoothness of a typical $2500 \AA$ film. (The thickness was determined by measuring the areal density of iron and assuming bulk density.) The pits revealed by the shadows are typically $500-800 \AA$ deep with evidence of shallower, longer range unevenness.

Since the films were about $2500 \AA$ thick, the samples closely approximate ideal twodimensional configurations. The two-dimensional solutions to the electrical current distributions were used without corrections for the finite thickness or observed roughness of the samples.

\subsection{Measurements}

The measurements were made at DC as a function of temperature in a gas flow cryostat (Bilger and Nicolet 1970). Temperatures were accurate to within $\pm 0.5 \mathrm{~K}$ and stable within $\pm 0.15 \mathrm{~K}$. Magnetoelectric effects were measured in fields of $0-23 \mathrm{kOe}$ with the field direction perpendicular to the plane of the sample. The field strength was set with an accuracy of \pm 10 Oe.

Resistivity was measured on the linear sample by supplying current at the ends and 

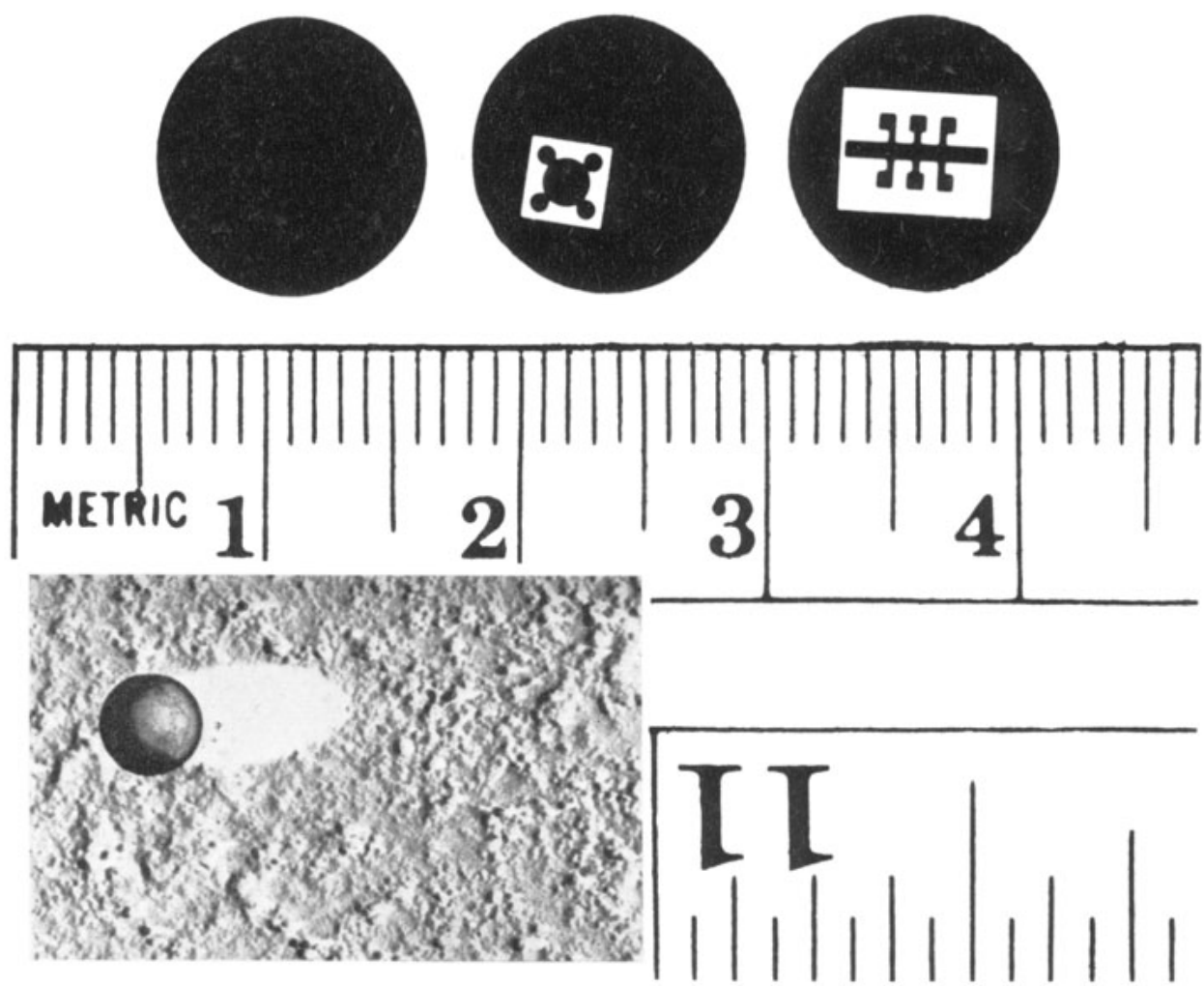

Figure 1. Macroscopic and microscopic views. Three typical $2500 \AA$ samples on glass substrates are shown next to a scale, The samples, from left to right, are a standard $1 \mathrm{~cm}$ diameter film, a 'van der Pauw' sample, and a linear sample, The inset shows a transmission electron micrograph of a carbon replica taken from a typical $2500 \AA$ film. The sphere is $5000 \AA$ in diameter. 
measuring the voltage across the sensing arms. The resistivity of the 'van der Pauw' sample was determined by supplying current through two adjacent contacts and measuring the voltage drop on the other pair; all eight possible current polarities and contact pairs were used to measure the resistivity and to determine the symmetry of the sample. The usual precautions were taken to minimize the effects of self-heating of the sample.

Hall effect measurements were performed on the 'van der Pauw' sample by applying the current to opposite contacts and measuring the voltage on the other pair. All eight possible combinations of current polarity, contact pairs, and magnetic field direction were used. Prior to each measurement, the sample was saturated in a field of $23 \mathrm{kOe}$. Then the field was reduced to zero and reversed, and measurements were performed at $10 \mathrm{Oe}, 4,10,23,10$, and $4 \mathrm{kOe}$. Then the field was again reversed and the measurements were repeated. The data for increasing field and decreasing field were analysed separately for evidence of magnetic hysteresis. More detailed measurements were performed at $104 \mathrm{~K}$ and $250 \mathrm{~K}$.

The transverse magnetoresistance measurements were made on the linear sample by directly measuring the change in the voltage drop as a function of the applied transverse magnetic field. The measurements were made in steps of $1 \mathrm{kOe}$ and with only one field direction.

The effect of the planar orientation of the magnetization on the magnetoresistance, or the planar Hall effect, was investigated by two-point and four-point measurements on the $1 \mathrm{~cm}$ samples. First, the voltage drop was measured in zero-field conditions. Then a magnetic field of 200 Oe or 450 Oe, larger than the coercive force, was applied parallel to the plane of the film to align the magnetization. The voltage drop was measured as a function of the orientation of the external field with respect to the current flow.

\section{Results and discussion}

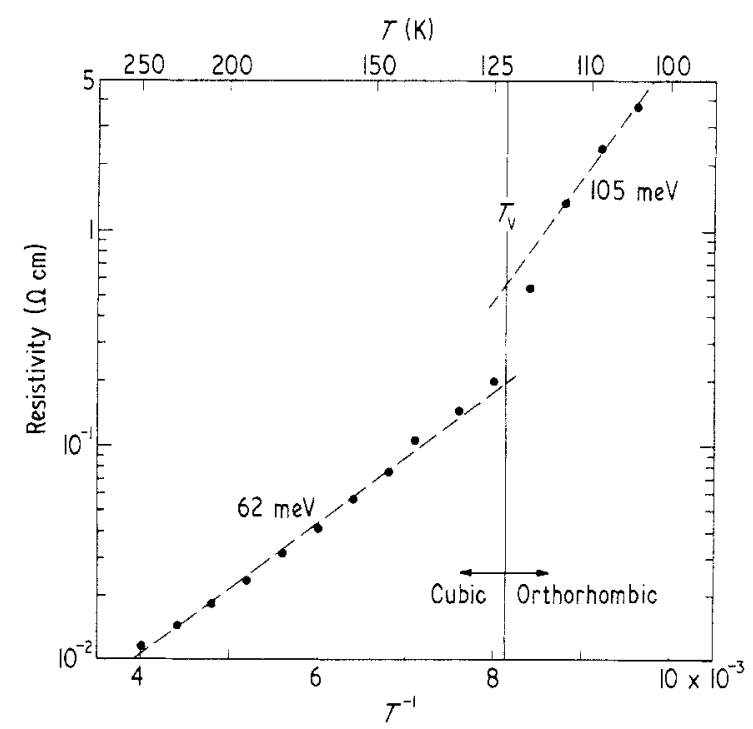

Figure 2. The resistivity of $2500 \AA \mathrm{Fe}_{3} \mathrm{O}_{4}$ on Corning 0211 glass as a function of temperature. The Verwey transition is clearly indicated but the change in the resistivity is not as large as in bulk samples. The transition also occurs at a slightly higher temperature than in bulk magnetite. These differences are due to substrate-induced stress. (Feugand and Nicolet 1975). 


\subsection{Resistivity}

The results of the resistivity measurements are shown in figure 2 . The room temperature resistivity is $8.5 \times 10^{-3} \Omega \mathrm{cm}$, or about $25 \%$ higher than the resistivity in bulk magnetite (Smit and Wijn 1959). We attribute this difference to the non-uniformity in the thickness of the films as shown in the inset in figure 1. The cubic phase has an activation energy of about $62 \mathrm{meV}$ while the orthorhombic phase has an activation energy of about $105 \mathrm{meV}$. Calhoun (1954) reported activation energies of $30 \mathrm{meV}$ and $110 \mathrm{meV}$, respectively. The difference in the conductivity and activation energies is not believed to be due to impurities in our samples. Impurity concentrations of less than $4 \%$ are known to increase the resistivity, but this also lowers the temperature of the Verwey transition (Epstein 1954).

The temperature of the Verwey transition was determined in our samples from more detailed resistivity measurements than shown in figure 2 . The transition temperature was taken as the midpoint between the two conductivity regions and was found to be $T_{\mathrm{V}}=123 \pm 1 \mathrm{~K}$, or about $4 \mathrm{~K}$ higher than reported for bulk magnetite (Calhoun 1954). We attribute these differences to stress imposed on the magnetite films by the substrate (Feng and Nicolet 1975).

\subsection{Hall effect}

The results of the Hall effect measurements are shown in figures 3,4 and 5. The polarities of both the ordinary and extraordinary Hall coefficients are negative at all temperatures.

The results of measurements taken in $1 \mathrm{kOe}$ steps at $250 \mathrm{~K}$ and $104 \mathrm{~K}$ are shown in figure 3 . The data for increasing and decreasing field at $250 \mathrm{~K}$ were analysed separately for evidence of magnetic hysteresis. Although there is a small systematic difference when the applied field is less than the saturation magnetization $\left(4 \pi M_{s}=6.0 \mathrm{kG}\right.$ at room temperature (Kittel 1966)), the largest difference corresponds to a difference in $4 \pi M_{\perp}$ of about $200 \mathrm{G}$. The differences for $H>4 \pi M_{\mathrm{s}}$ are due to the errors of measurement. The data for increasing and decreasing field at $104 \mathrm{~K}$ were averaged together since the uncertainties in the measurements were too large to resolve any hysteresis.

The results clearly indicate the presence of two distinct régimes. The low-field signal $\left(H<4 \pi M_{\mathrm{s}}\right)$ is due primarily to the extraordinary Hall effect while the nonzero slope in the high-field region is due solely to the ordinary Hall effect. The intersection of the asymptotes to these two sections is at about $5-6 \mathrm{kOe}$ as expected from the saturation magnetization and the demagnetization factor of nearly unity.

At other temperatures, the extraordinary Hall coefficient was determined from the Hall voltages measured at $10 \mathrm{Oe}$ and $\pm 4 \mathrm{kOe}$ and the ordinary Hall coefficient was determined from the difference of the Hail voltages at $10 \mathrm{kOe}$ and $23 \mathrm{kOe}$. These were chosen as a compromise between signal amplitudes and errors. The small departure at $4 \mathrm{kOe}$ from the asymptote corrects, in part, for the fact that the Hall voltage below saturation is due to both the ordinary and extraordinary Hall coefficients.

4.2.1. Extraordinary Hall effect. The results of the extraordinary Hall effect measurements as a function of temperature are shown in figure 4 where $X$ is the room temperature value reported by Lavine (1959). It is in reasonable agreement with the present results. The activation energies are about $26 \mathrm{meV}$ in the cubic phase and $95 \mathrm{meV}$ in the orthorhombic phase. 


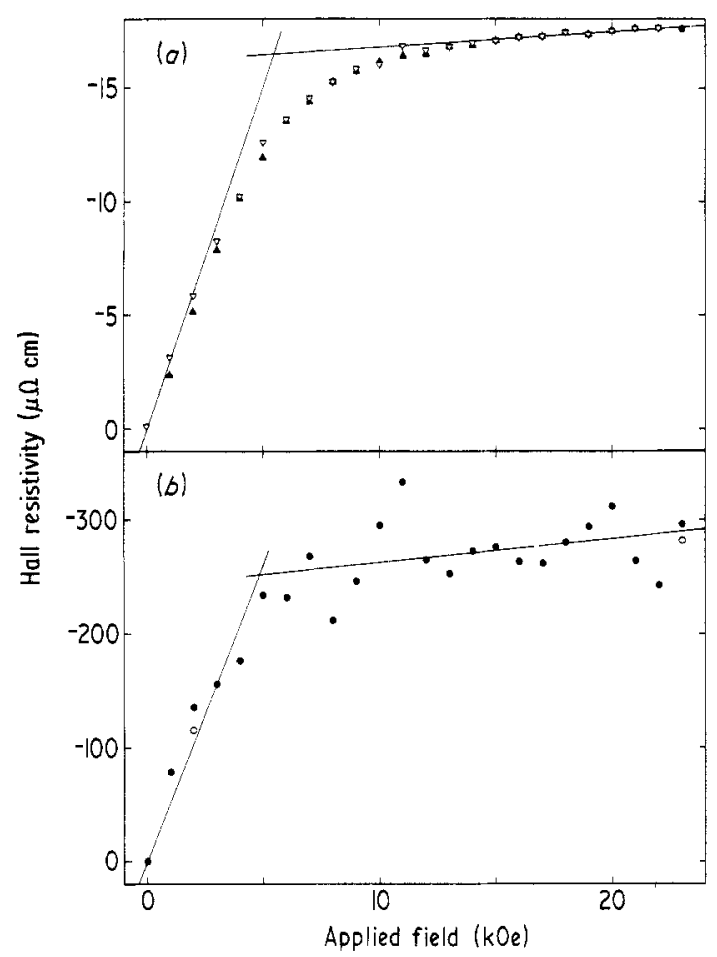

Figure 3. Hall resistivity of $2500 \AA \mathrm{Fe}_{3} \mathrm{O}_{4}$ on Corning 0211 glass as a function of the applied magnetic field. (a) The Hall resistivity at $250 \mathrm{~K}$. The data for increasing and decreasing field were analysed separately and they reveal the existence of a small magnetic hysteresis below saturation. The ordinary and extraordinary Hall coefficients are both negative: $\boldsymbol{\Lambda}$, increasing field; $\nabla$, decreasing field; - maximum field $=23 \mathrm{kOe}$. $(b)$ The Hall resistivity at $104 \mathrm{~K}$. There is no evidence of polarity reversal as reported by Kostopoulos and Theodossiou (1970). The scatter in the data points is due to temperature variations of about $\pm 0.1 \mathrm{~K}$ : - $15 \mu \mathrm{A} ; \mathrm{O}, 5 \mu \mathrm{A}$.

As stated in $\$ 2.1$, calculations predict that the extraordinary Hall coefficient should have a square law dependence on the resistivity for metallic d-band conduction. The extraordinary Hall coefficient in figure 4 as a function of the resistivity (figure 2) is shown in figure 5. The best power law fit to the data is a $\rho^{1 / 3}$ dependence above $T_{\mathrm{V}}$ and $\rho^{2 / 3}$ below $T_{\mathrm{V}}$. A re-examination of Lavine's (1961) data also produces a $\rho^{1 / 3}$ dependence over the same temperature range.

4.2.2. Ordinary Hall effect. The ordinary Hall coefficient as a function of temperature is also shown in figure 4 . Observe that the scales of the two ordinates differ by a factor of 100. The activation energies of about $22 \mathrm{meV}$ above $T_{\mathrm{V}}$ and about $100 \mathrm{meV}$ below $T_{\mathbf{V}}$ are consistent with the results of Siemons (1970). However, an important difference is that our results indicate a negative Hall coefficient while Siemons reported a positive Hall coefficient. Siemons attributed part of the Hall voltage at high magnetic fields to the extraordinary Hall effect. The saturation magnetization in his sample varied by about $1 \%$ in an external field varying from $10 \mathrm{kHe}$ to $15 \mathrm{kOe}$. This was claimed to have added an extraordinary Hall signal proportional to the change in the magnetization. 


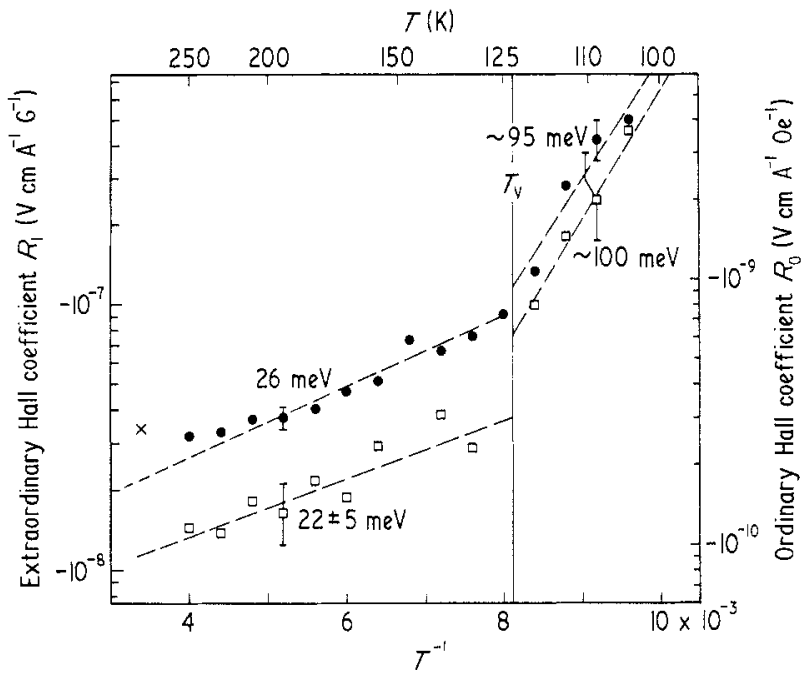

Figure 4. The ordinary and extraordinary Hall coefficients as a function of temperature. Note that there is almost a factor of 100 difference between the two. Also shown for comparison is the value of $R_{1}$ (marked $x$ ) reported by Lavine (1959). Note that the ratio of the ordinary and extraordinary Hall coefficients is approximately constant in both the cubic and orthorhombic phases. The error bars shown are estimates of the typical errors above and below $T_{V}:$, extraordinary Hall coefficient; $₫$, ordinary Hall coefficient.

After making the correction for this effect, Siemons calculated a net positive Hall coefficient. In our case, the observed ordinary Hall signal is about $10 \%$ of the extraordinary Hall voltage. Thus, even if this correction were applied, it would only reduce the ordinary Hall coefficient by $10-20 \%$, but it would not change the sign of the observed ordinary Hall coefficient. Since the high-field dependence of $4 \pi M_{\perp}$ (for $H_{\perp}>4 \pi M_{s}$ ), if any, is not known in our samples, we did not apply any such corrections to our data.

One result of the present data is that the ratio of the ordinary and extraordinary Hall

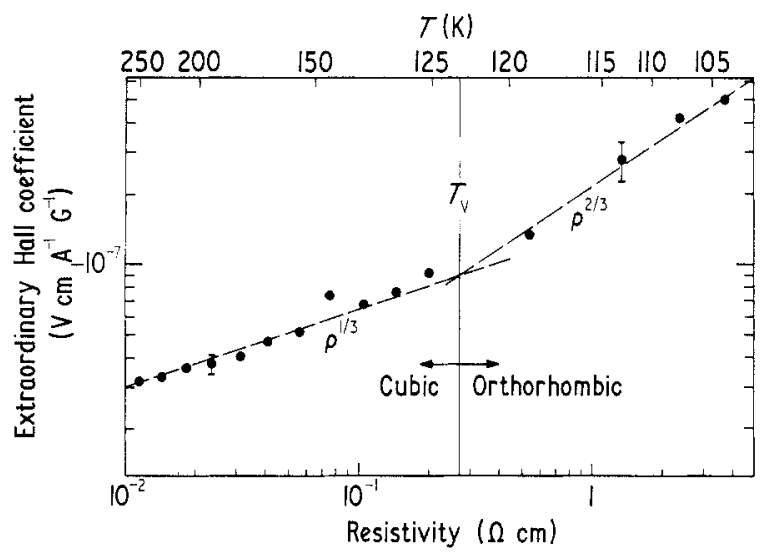

Figure 5. The extraordinary Hall coefficient as a function of the resistivity. The best power law fits to these data are a $\rho^{1 / 3}$ dependence in the cubic phase and $\rho^{2 / 3}$ below $T_{\mathrm{V}}$. The two error bars shown are estimates of the typical errors above and below $T_{\mathbf{v}}$. 
coefficients is approximately independent of the temperature and the ratio is only slightly different in the two phases.

4.2.3. Hall mobility. The Hall mobility can be evaluated from the ordinary Hall effect and resistivity data using the formula

$$
\mu_{\mathrm{H}}=10^{8} \times \frac{R_{0}}{\rho}
$$

where $R_{0}$ is in $\mathrm{Vcm}^{-1} \mathrm{Oe}^{-1}, \rho$ is in $\Omega \mathrm{cm}$, and $\mu_{\mathrm{H}}$ is in $\mathrm{cm}^{2} \mathrm{~V}^{-1} \mathrm{~s}^{-1}$. The mobility as a function of temperature is shown in an Arrhenius plot in figure 6. Above $T_{\mathrm{V}}$ the mobility

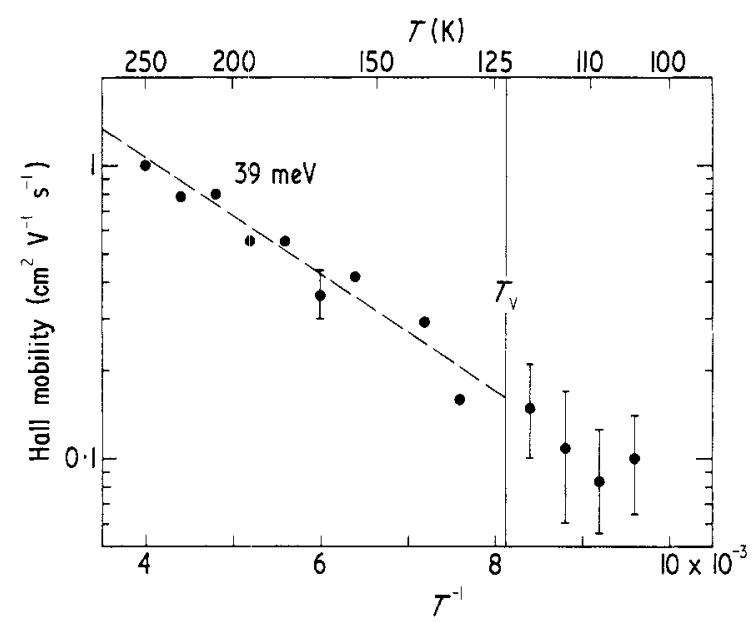

Figure 6. Hall mobility as a function of temperature. The positive temperature coefficient in the cubic phase is evidence of hopping conductivity. The error bar shown above $T_{v}$ is an estimate of the errors typical for the cubic phase. The error bars shown below $T_{\mathrm{v}}$ indicate that the present data are insufficient to clearly reveal a temperature dependence for the orthorhombic phase.

has a positive temperature coefficient with an activation energy of about $39 \mathrm{meV}$. Although the Hall mobility data show no definite temperature dependence below $T_{\mathrm{V}}$, the measurements were confined to $104-120 \mathrm{~K}$ and cannot be considered conclusive. The best fit of a power law dependence to the present mobility measurements varies from about $T^{4 \pm 1}$ at low temperatures to about $T^{3 \pm 1}$ near room temperature.

\subsection{Magnetoresistance}

4.3.1. Dependence on the orientation of the magnetization. Magnetoresistance is an even function of both the transverse and longitudinal components of the magnetic field. If the magnetoresistance is a function of the magnetization, it must be measured with the magnetization both parallel and perpendicular to the current. Since the in-plane demagnetizing factors are small, the magnetization can be oriented in the plane with small-bias fields. Any magnetoresistance observed as a function of the position of the magnetization in the plane, or planar Hall effect, will be due primarily to the magnetization since the required bias fields will be much smaller than the magnetization.

We have performed such measurements by two-point and four-point measurements on $1 \mathrm{~cm}$ diameter samples. The orientation of the magnetization in the plane of the film 
was established using planar bias fields of $200 \mathrm{Oe}$ and $450 \mathrm{Oe}$. The apparent resistivity did not change measurably when the magnetization was rotated. We therefore conclude that the upper limit on the magnetoresistance due to the magnetization is $\leqslant 0.2 \%$ at room temperature.

4.3.2. Dependence on the transverse applied field. The transverse magnetoresistance was measured as a function of both the applied field and the temperature. The change in the voltage drop in the linear sample, normalized to the zero-field voltage drop, is shown in figure 7 as a function of the applied field at $250 \mathrm{~K}$. The magnetoresistance was

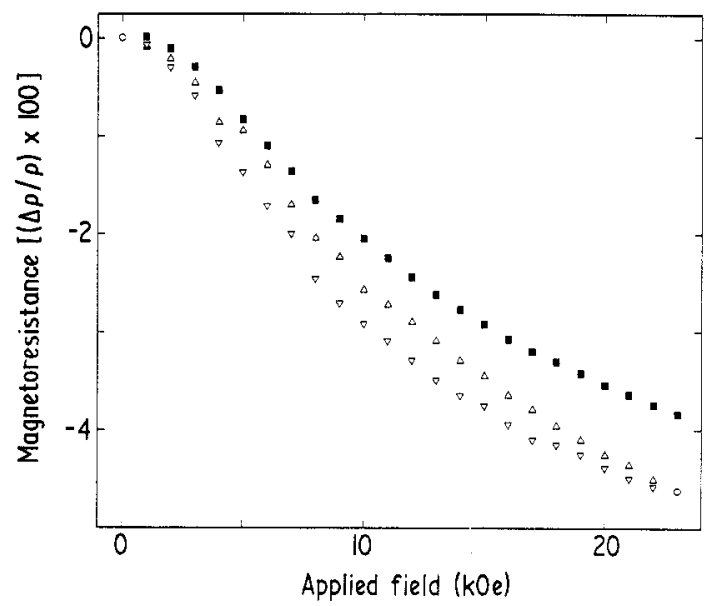

Figure 7. Transverse magnetoresistance as a function of the applied field at $250 \mathrm{~K}$. The magnetoresistance is negative for all magnetic field strengths. The data for increasing and decreasing magnetic field on the 'van der Pauw' sample (open triangles) are shown separately to reveal the hysteresis : $\Delta$, low field to high field; $\nabla$, high field to low field. The data from the linear samples $(\square)$ were taken with increasing field. The magnetoresistance is quadratic at low fields and does not saturate or become linear at applied fields up to $23 \mathrm{kOe}$. This relative field dependence is typical for all temperatures investigated. Simultaneous Hall effect measurements (figure 3) give clear evidence of the saturation of the perpendicular component of the spontaneous magnetization.

also determined by analysing the Hall effect data from the 'van der Pauw' sample with the induced voltage normalized to the offset voltage caused by the asymmetry in the position of the contacts. These results are also shown in figure 7. A comparison shows that the two methods agree within $15-30 \%$, but the magnitude of the results from the 'van der Pauw' sample are systematically larger than those from the linear sample. The magnetoresistance is negative and quadratic at low magnetic fields. There is an inflection point in the field dependence near saturation and the magnitude increases monotonically to about $4 \%$ at a magnetic field of $23 \mathrm{kOe}$. This type of qualitative field dependence is typical for all temperatures investigated.

The results of the transverse magnetoresistance measurements at $4 \mathrm{kOe}, 10 \mathrm{kOe}$, and $23 \mathrm{kOe}$ are shown in figure 8, as a function of temperature. The error bars shown in 23 kOe points in the 'van der Pauw' sample are typical of the errors for all measurements made at that temperature on those samples. The estimated error in the data taken on the linear sample is about $\pm \frac{1}{2} \%$ for all points with the errors increasing slightly at lower temperature. The data show that the high-field magnetoresistance has an extremum 
in the temperature dependence near the Verwey temperature and that it is negative for all temperatures and field strengths. The magnetoresistance reaches $7-7 \frac{1}{2} \%$ at about $130 \mathrm{~K}$ and $23 \mathrm{kOe}$.

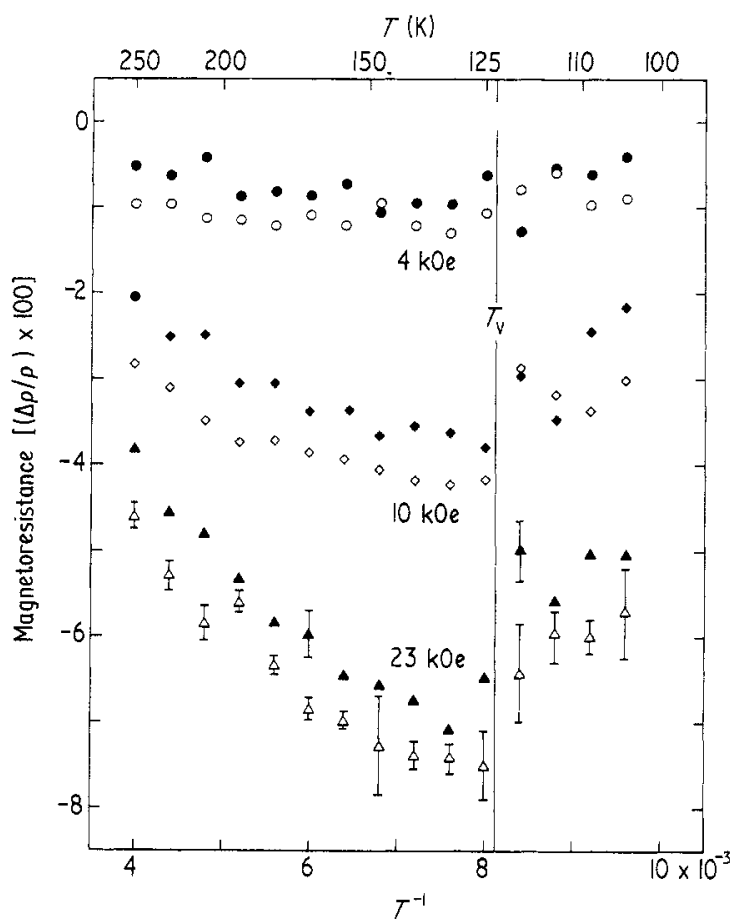

Figure 8. The transverse magnetoresistance as a function of temperature. The magnetoresistance is negative at all temperatures and all magnetic field strengths investigated. The data shown are for both the linear sample (full symbols) and the 'van der Pauw' samples (open symbols) at $4 \mathrm{kOe}$ (open and full circles), $10 \mathrm{kOe}$ (open and full diamonds) and $23 \mathrm{kOe}$ (open and full triangles). The error bars shown on the $23 \mathrm{kOe}$ data from the 'van der Pauw' sample are also applicable to the data taken at the same temperature but at lower magnetic fields. The two error bars shown for the linear sample are estimates of the typical errors above and below $T_{\mathrm{V}}$.

The present transverse magnetoresistance results differ significantly from those previously reported. The magnitude of the magnetoresistance in our measurements increases monotonically with the magnetic field at all temperatures. It is the largest ever observed in cubic magnetite; it does not exhibit any change in sign; and it is quadratic at low magnetic fields and does not saturate with the magnetization. The magnetoresistance has its maximum magnitude at about $130 \mathrm{~K}$ and decreases at higher and lower temperatures.

The present measurements also separate the effects of the applied field and the spontaneous magnetization on the transverse magnetoresistance. At room temperature, it is apparently dominated by the externally applied field and almost unaffected by the magnetization. An alternative explanation is that the transverse and longitudinal magnetoresistances due to the magnetization could have the same magnitude. The differences in these interpretations cannot be tested experimentally.

We suspect that the general lack of agreement on the transverse magnetoresistance of the cubic phase (Domenicali 1950, Zalesskii 1961, Kostopoulos 1970) is due to im- 
purities in the samples since most of the previously reported results were taken on natural magnetite crystals. The present measurements were performed on synthetic samples whose impurity content is believed to be low. We believe that the essential qualitative features of our result will be reproduced on synthetic, single crystal samples. Note however that our samples are polycrystalline and the results are therefore averages over all crystallographic directions.

The results below $T_{\mathrm{V}}$ agree qualitatively with those of Kostopoulos (1972) despite the differences in the results above $T_{\mathrm{v}}$. This suggests that the low-temperature magnetoresistance may be dominated by the structure and the change in the specific resistivity will be approximately independent of the sample.

\section{Conclusions}

The results are summarized in table 1 . The resistivity measurements demonstrate that the properties of thin films of magnetite are basically similar to those of bulk magnetite. Both forms exhibit a thermally activated conductivity and the conductivity transition

Table 1.. Galvanomagnetic properties of magnetite thin films.

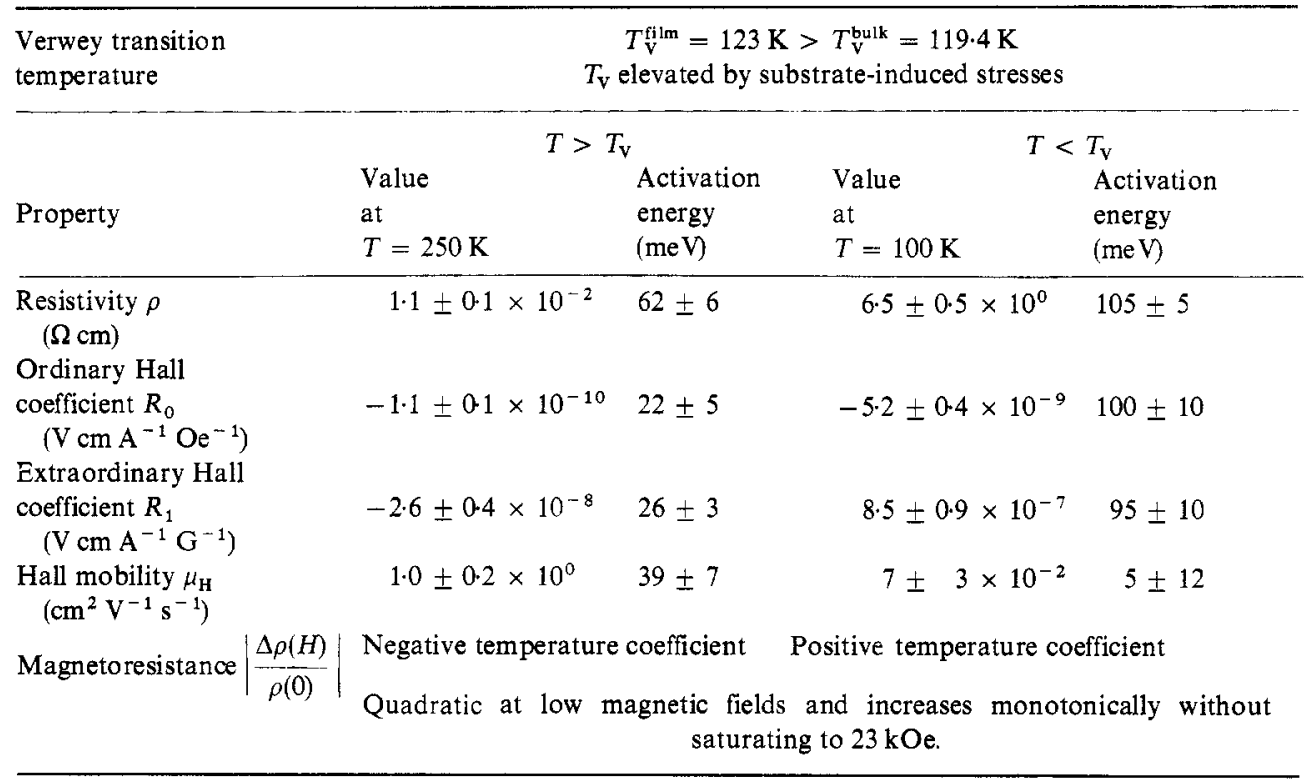

is present in both cases. The different activation energies, higher Verwey transition temperature, and smaller resistivity change at the transition observed in the thin films are unique to thin film samples.

The extraordinary Hall coefficient $R_{1}$ has been shown to have a power law dependence on the resistivity. The best fit to the present results indicates that $R_{1}$ is proportional to $\rho^{1 / 3}$ in the cubic phase and $\rho^{2 / 3}$ in the orthorhombic phase.

The ordinary Hall coefficient is negative. This is usually interpreted as a result of electronic conductivity.

While the Hall effect, a first-order phenomenon in the magnetic field, is dominated 
by the spontaneous magnetization, the magnetoresistance, a second-order effect due to the magnetic field, is apparently insensitive to the magnetization.

The present measurements show that the transverse magnetoresistance due to an externally applied field is negative at all temperatures and field strengths. It is quadratic at low magnetic fields and it increases monotonically without saturating up to $23 \mathrm{kOe}$. The magnitude of the magnetoresistance is large in magnetite and is of the order of $2-4 \%$ at $10 \mathrm{kOe}$.

These results illustrate some of the advantages and disadvantages of performing galvanomagnetic measurements on thin films of ferromagnetic materials. Ferromagnetic films have demagnetizing factors of almost exactly unity. In the absence of crystalline or perpendicular anisotropies, the perpendicular components of the magnetic field are simply determined by knowing the applied field, and the effects of the applied field and the spontaneous magnetization can be easily separated. The electrical current and potential distributions are easily determined from the geometry and two-dimensional potential theory with negligible corrections for the finite thickness of the samples. Since the samples are synthetic, the composition should be well established. The potential disadvantages are the polycrystallinity of the samples and the possibility of anomalous results due to the presence of a substrate. It may be possible to produce epitaxial single crystal films. However, measurements on polycrystalline samples obviously reflect averages over all possible crystallographic directions. The presence of a mechanical substrate-sample interaction can be minimized by a judicious choice of substrates. However, it can also be used advantageously to study the effect of large amounts of tensile stress on the properties of a material.

\section{Acknowledgments}

This work would not have been possible without the cooperation of J L Beauchamp, who kindly allowed us to use his electromagnet. We are also indebted to F B Humphrey, $\mathrm{T} \mathrm{M}$ Morris, and $\mathrm{C} \mathrm{H}$ Wilts for the use of their facilities and for guidance. We thank C H Bajorek (IBM-Thomas $\mathbf{J}$ Watson Research Center) for providing the electron micrograph and for helpful discussions. Thanks are also due to T C McGill for stimulating conversations on electronic transport in solids.

\section{References}

Berger L 1972 Phys. Rev. B 5 1862-70

Bickford L R 1953 Rev. Mod. Phys. 25 75-9

Bilger H R and Nicolet M-A 1970 Rev. Sci. Instrum. $41346-7$

Calhoun B A 1954 Phys. Rev. 94 1577-85

Constantin C and Rosenberg M 1972 Proc. 18th Conf. on Magnetic Materials (New York: AIP) 10 $1389-92$

Domenicali C A 1950 Phys. Rev. 78 458-67

Drabble J R. Whyte T D and Hooper R M 1971 Solid St. Commun. 9 275-8

Epstein J H 1954 PhD Thesis MIT

Feng J S-Y, Bajorek C H and Nicolet M-A 1972 IEEE Trans. Magnetics MAG-8 277-8

Feng J S-Y and Nicolet M-A 1975 J. Phys. Chem. Solids (in press)

Heikes R R and Johnston W D 1959 J. Chem. Phys. 26 582-7

Humphrey F B 1967 J. Appl, Phys, 38 1520-7

Karplus R and Luttinger J M 1954 Phys. Rev. 95 1154-60

Kittel C 1966 Introduction to Solid State Physics (New York: Wiley) p 461

Kostopoulos D 1972 Phys. Stat. Solidi (a) $9523-7$ 
Kostopoulos D and Theodossiou A 1970 Phys. Stat. Solidi (a) 2 73-8

Lavine J M 1959 Phys. Rev. 114 482-8

1961 Phys. Rev. 123 1273*7

Li C H 1932 Phys. Rev. 40 1002-12

Millar R W 1929 J. Am. Chem. Soc. 51 215-22

Pugh E M and Rostoker N 1953 Rev. Mod. Phys. 25 151-7

Putley E H 1968 The Hall Effect and Semiconductor Physics (New York: Dover) p 18

Siemons W J 1970 IBM J. Res. Dev. 14 245-7

Smiltens J 1952 J. Chem. Phys. 20 990-4

Smit J and Wijn H P J 1959 Ferrites (New York: Wiley) p 229

Turner C E 1972 J. Phys. C: Solid St. Phys. 5 2859-66

van der Pauw L J 1958 Philips Res. Rep. $131-9$

1959 Philips Tech. Rev. $20220-4$

Verwey E J W, Haayman P W and Romeijn F C 1947 J. Chem. Phys. 15 181-7

Weiss $P 1896$ PhD Thesis University of Paris

Zalesskii A V 1961 Sov. Phys.-Crystallogr. 6 180-5 